\title{
Fonctionnement des quilles de voiliers
}

\section{Christian Jacquot}

Assistant à l'Ecole Nationale Supérieure de Mécanique de Nantes

\section{Dominiaue Rajaona}

Ingénieur de Recherches au Laboratoire d'Hydrodynamique Navale de l'E.N.S.M.

La méthode présentée a pour but de mettre en évidence l'influence des paramètres de gite, de flèche et d'allongement de la quille du voilier. Le calcul théorique ainsi que des mesures en bassin des carènes et en soufflerie, ont permis de déterminer les efforts et moments exercés par le fluide sur la quille, pour différents points de fonctionnement.

\section{Introduction}

Le fonctionnement d'un voilier est un phénomène complexe qui fait intervenir des facteurs aussi différents que l'hydrodynamique de la carène, l'aérodynamique de la voile, l'état de la mer, la qualité de l'équipage ou la reglementation de la course. Le problème ne peut évidemment être envisagé sous tous ces aspects et certains des facteurs cités peuvent difficilement être pris en compte dans des équations. Cependant, une bonne conception de l'hydro et aérodynamique du bateau sera toujours indispensable.

Même en se limitant au seul aspect hydrodynamique $\mathrm{du}$ fonctionnement de la carène, les études restent compliquées et demandent des moyens dont ne dispose pas la construction de plaisance. Les études entreprises ont rarement un caractère fondamental, elles consistent généralement en des essais de remorquages d'une maquette dont les plans ont été tracés par un architecte naval. La conception des plans résulte de l'expérience accumulée aussi bien au cours des essais en bassin qu'en cours de croisière ou régate en mer. Les carènes ainsi conçues sont souvent de très bonne qualité, mais il est difficile, peu d'essais systématiques ayant été faits, de déterminer les raisons qui font que le choix d'une solution a été meilleur qu'une autre.
Pour toutes ces raisons, il nous a paru intéressant d'aborder l'étude théorique et expérimentale présentée ici, et d'en tirer les conclusions pour une meilleure compréhension des phénomènes physiques mis en jeu.

\section{Méthode théorique}

\section{Formulation du problème}

On se propose d'étudier l'écoulement tridimensionnel autour d'une quille de voilier placée en gîte et dérive et animée d'un mouvement uniforme dans le fluide (fig. 1).

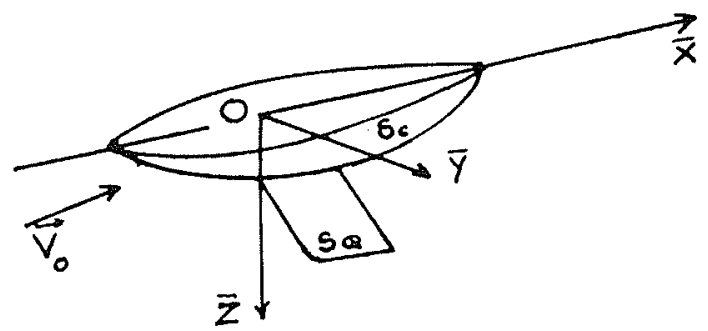

Figure 1

Soient :

- $S_{C}$ la surface de la carène

- $S_{Q}$ la surface de la quille

- $\vec{n}$ la normale extérieure à $S_{C}$ et $S_{Q}$

- $\alpha$ l'angle de dérive

- $\beta$ l'angle de gîte

- Le trièdre $O \bar{X} \bar{Y} \bar{Z}$ est le repère lié au voilier

- $\vec{V}_{0}$ l'écoulement incident. 


\section{Hypothèses}

$\left|H_{\text {I }}\right|$ Le fluide est parfait isovolume

$\left|H_{2}\right|$ La surface libre supposée suffisamment peu déformée, est assimiliée au plan. L'écoulement est donc le même que celui considéré en prenant la carène et son image par rapport à ce plar dans un fluide illimité. L'ensemble de la carène et de son image constitue le double modèle.

$\left|H_{3}\right|$ L'effet de portance de la quille entraine l'existence d'une nappe de tourbillons libres $(\Sigma)$ issus de son bord de fuite. Cette nappe a pour support les lignes de courant de l'écoulement sans portance.

Comme il est d'usage dans les théories des surfaces portantes, le sillage est plan, sans épaisseur et reste dans le plan de symétrie de la quille.

$\left|H_{4}\right|$ L'angle de dérive $\alpha$ est en pratique faible pour les conditions habituelles de navigation. Nous étudierons les incidences comprises entre 0 et $10^{\circ}$.

${ }_{1} H_{5} \mid$ L'angle de gîte $\beta$ est défini par l'angle de rotation $\mathrm{du}$ voilier autour de son axe longitudinal $\beta \leqslant 30^{\circ}$.

$\left\{H_{6} \mid\right.$ La carềne $S_{C}$ n'engendre pas de tourbillons libres. Cela revient à supprimer le bustle et le gouvernail.

\section{Mise en équation}

1 - La résolution de l'équation de continuité conduit au problème de la recherche d'un potentiel harmonique $\phi_{e}$ dans le domaine $\mathcal{O}_{e}$ extérieur à $S_{C} U S_{Q} U \Sigma$.

La méthode que nous utilisons pour le résoudre est la méthode des singularités [1] [2] [3] [4] [5] [7].

Le potentiel des vitesses est généré par deux types de singularités :

- des sources réparties sur la surface de l'ensemble quille-carène ;

- des doublets sur le sillage et la quille.

La fermeture des tourbillons libres et liés du sillage et de la quille est assurée par une nappe de doublets située à l'intérieur de la carène. Cette nappe est obtenue en prolongeant les doublets à l'emplanture de la quille.

Le potentiel induit en $M$ par l'ensemble des singularités considéré, s'écrit alors :

$\varphi(M)=(-1) / 4 \Pi \int_{S_{C} U S_{Q}} \sigma\left(M^{\prime}\right)-1 /\left|\overrightarrow{M M}^{\prime}\right| d S_{M^{\prime}}$
$+(-1) / 4 \Pi \int_{S_{Q} U \Sigma} \mu\left(M^{\prime}\right) \partial / \partial n_{M^{\prime}}\left(1 /\left|M \vec{M}^{\prime}\right|\right) d S_{M^{\circ}}(1)$

2 - Données aux limites

2.1. - Le fluide glisse sur la surface $S_{C} U S_{Q}$. La composante normale de la vitesse absolue est donc nulle en tout point $M$ de $S_{C} U S_{Q}$ :

$\partial \phi / \partial n=0 \quad \partial \phi / \partial n=-\vec{V}_{0} \cdot \vec{n}_{M} \quad \forall M \in S_{Q} U S_{C}$

2.2. .. La condition de Joukowski est satisfaite si le fluide ne contourne pas le bord de fuite de la quille. Pour cela, on fait l'hypothèse que le fluide glisse sur le plan de symétrie de la quille dès qu'il quitte son bord de fuite ; ainsi, il revient au même d'écrire une condition de glissement du fluide sur une ligne $\delta(\delta \in \Sigma)$ parallèle au bord de fuite et située à une distance très faible de ce dernier. Définissons $\vec{n}_{\Sigma}$ la normale au sillage en chaque point de $\delta$. On a :

$$
\partial \phi / \partial n=-\vec{V}_{0} \cdot \vec{n}_{M} \quad \forall M \in \delta
$$

\section{3 - Equation intégrale du problème}

3.1 - L'ensemble de ces données aux limites se traduit par l'équation intégrale :

$\lambda \sigma(M) / 2-$

$-\int_{S_{C} U S_{Q}} \sigma\left(M^{\prime}\right) / 4 \Pi \cdot \partial / \partial n_{M} \cdot\left(1 /\left|\overrightarrow{M M}^{\prime}\right|\right) d S_{M^{\prime}}$
$-\int_{S_{Q} U \Sigma} \mu\left(M^{\prime}\right) / 4 \Pi \cdot \partial / \partial n_{M} \cdot\left(\partial / \partial n_{M^{\prime}}(1 / / M \vec{M} \mid)\right) d S_{M^{2}}$
$=-\vec{V}_{0} \cdot \vec{n}_{M}$

avec :

$$
\begin{array}{lll}
\lambda=1 & \text { si } & M \in S_{C} U S_{Q} \\
\lambda=0 & \text { si } &
\end{array}
$$

3.2. -- Introduisons une seconde forme de l'équation intégrale (4) qui permet de réduire le nombre des inconnues du problème discrétisé, comme nous le verrons plus loin.

La non unicité de la répartition des singularités dans la génération d'un potentiel nous autorise à faire un choix sur la distribution de doublets $\mu\left(M^{\prime}\right)$ sur $S_{Q^{\prime}}$ à condition toutefois, de respecter la continuité du potentiel au raccordement de $S_{Q}$ et $\Sigma$.

De plus, sur $\Sigma, \mu\left(M^{\prime}\right)$ est égale à la circulation changée de signe.

Dans ces conditions, pour chaque section placée à $z$ constant de $S_{Q} U \Sigma$, on peut introduire une fonction $F$ connue telle que :

$$
\mu\left(M^{\prime}\right)=-F \Gamma\left(z_{M^{\prime}}\right)
$$

De cette façon $\mu$ est connue dès la détermination de $\Gamma\left(z_{M^{\prime}}\right)$. Pour assurer la continuité de $\mu\left(M^{\prime}\right)$ au bord de fuite, on choisit une fonction linéaire sur les deux faces de la quille. Soit :

$$
F \begin{cases}F(\gamma)=F^{+}(\nu)=1 / 2 & M^{\prime} \in S_{Q}^{+} \\ F(\gamma)=F^{-}(\nu)=-1 / 2 & M^{\prime} \in S_{Q}^{-} \\ F(\gamma)=1 & \end{cases}
$$

$\nu$ étant défini comme l'abscisse réduite de $M^{\prime}$ en prenant le bord d'attaque local comme origine :

$$
\nu=\left(x\left(M^{\prime}\right)-x_{B A}\right) / C_{l}\left(z_{M^{\prime}}\right)
$$

$x_{M^{\prime}}$ abscisse de $M^{\prime}$ dans $O \bar{X} \bar{Y} \bar{Z}$

$x_{B A}$ abscisse du bord d'attaque local du profil de la section à la cote $z$

$C_{l}\left(z_{M}\right)$ corde locale. 


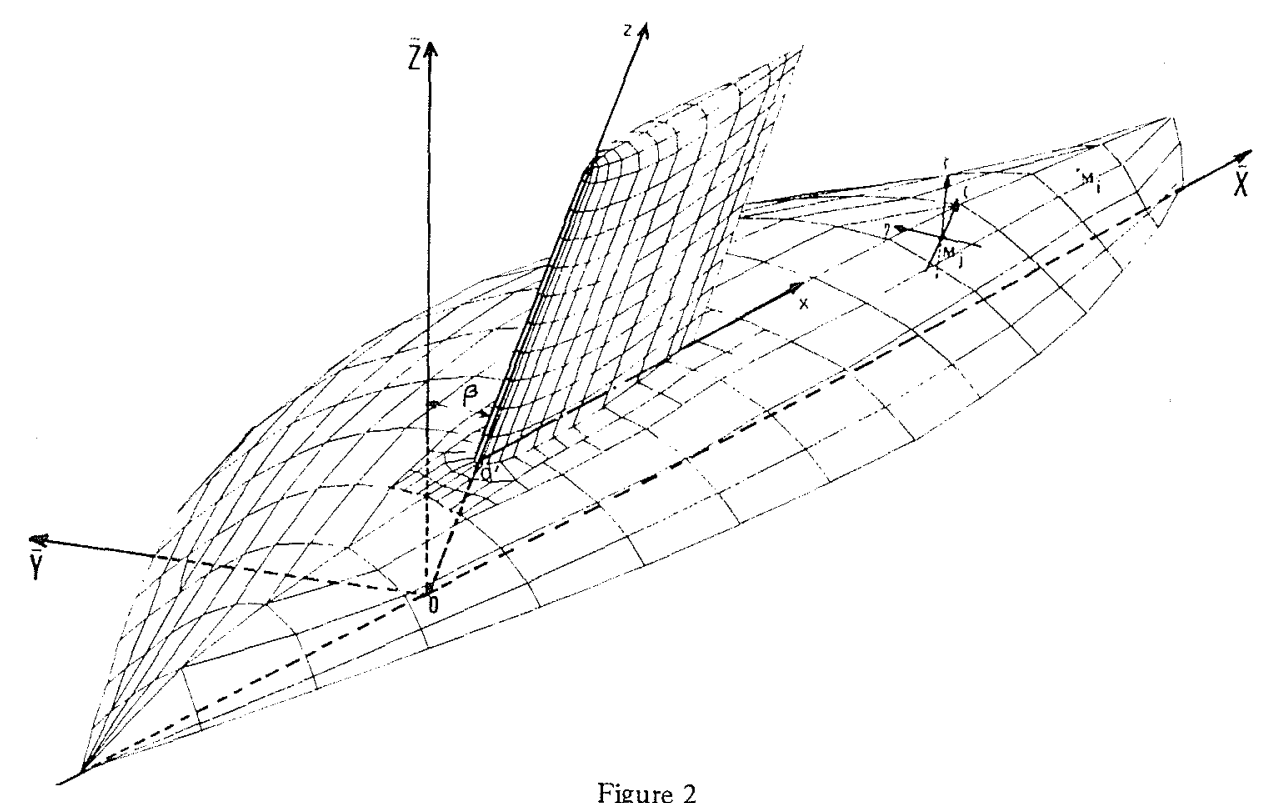

L'équation intégrale (4) devient alors :

$\lambda \sigma(M) / 2$

$$
\begin{array}{r}
-\int_{S_{C} U S_{Q}} \sigma\left(M^{\prime}\right) / 4 \Pi . \partial / \partial n_{M}\left(1 /\left|M M^{\prime}\right|\right) d S_{M^{\prime}} \\
+\int_{S_{Q} U \Sigma} F \cdot \Gamma \partial / \partial n_{M}\left(\partial / \partial n_{M^{\prime}}\left(1 /\left|M M^{\prime}\right|\right)\right) d S_{M^{\prime}} \\
=-\vec{V}_{o} \cdot \overrightarrow{n_{M}}
\end{array}
$$

\section{Principe de la méthode de résolution}

L'équation intégrale (8) est remplacée par une somme finie. La surface du double modèle est approchée par un ensemble de facettes planes élémentaires à trois ou quatre côtés. A chaque figure élémentaire on associe un point de contrôle où sera affichée la condition de glissement (Fig. 2).

La distribution de singularités est supposée constante sur chaque facette.

Dans ces conditions, on peut remplacer les intégrales de (8) par :

$$
\begin{aligned}
\sum_{j=1}^{N_{S}} \sigma_{j} \int_{\Sigma e_{j}} K\left(M_{i}, M^{\prime}\right) d S_{M^{\circ}}+\sum_{j=1}^{N_{k}} \Gamma_{j} \\
\int_{S B_{j}} L\left(M_{i^{\prime}} M^{\prime}\right) d S_{M^{\prime}}=-\vec{V}_{o} \vec{n}_{M_{i}}
\end{aligned}
$$

où $\Sigma e_{j}$ : surface de la facette élémentaire

$S B_{j}$ : surface de la bande de la quille et du sillage.

$K\left(M, M^{\prime}\right)$ : noyau de l'équation intégrale relatif aux sources

$L\left(M, M^{\prime}\right)$ : noyau de l'équation intégrale relatif aux doublets.

L'expression matricielle du système linéaire (9) est :

$$
\left[\begin{array}{c:cc}
A_{i j} & B_{i j} \\
& &
\end{array}\right]\left[\begin{array}{c}
\sigma_{i} \\
\Gamma_{j}
\end{array}\right]=-\left[\begin{array}{l}
V_{o} n_{i} \\
\end{array}\right]
$$

où :

$$
\begin{aligned}
& A_{i j}=\int_{\Sigma e_{j}} K\left(M_{i}, M^{\prime}\right) d S_{M^{\circ}} \\
& B_{i j}=\int_{S B_{j}} L\left(M_{i}, M^{\prime}\right) d S_{M^{\prime}}
\end{aligned}
$$

et :

$$
\begin{aligned}
& K\left(M_{i}, M^{\prime}\right)=\partial / \partial \eta_{M i}\left(1 /\left|\overrightarrow{M_{i} M^{\prime}}\right|\right) \text { et } \\
& L\left(M_{i}, M^{\prime}\right)=\partial / \partial \eta_{M_{i}}\left(\partial / \partial \eta_{M},\left(1 /\left|\overrightarrow{M_{i} M^{\prime}}\right|\right)\right.
\end{aligned}
$$

La matrice $\left|A_{\ddot{i}}\right|$ est la matrice des coefficients d'influence des facettes de sources sur les points de contrôle ; la partie carrée de $\left|A_{i j}\right|$ correspond à la matrice du problème non portant (obtenue en annulant $\mu$ et en supprimant les points de contrôle "Joukowski").

Les éléments de la matrice $\left|B_{i j}\right|$ sont les coefficients d'influence d'une bande attachée à l'inconnue $\Gamma_{j}$; de ce fait elle n'a pas la même nature que $\left|A_{i j}\right|$ et se calcule différemment. En effet, chaque $B_{i j}$ est la somme de coefficients d'influence de doublets répartis sur des facettes de la quille et sur la lanière tourbillonnaire qui s'en échappe.

Le système matriciel $(10)$ est résolue par la méthode itérative de Gauss-Siedel ; cette méthode, bien adaptée au problème, permet de résoudre les systèmes de grande dimension sans prendre trop de place en mémoiremachine.

\section{Exploitation du programme de calcul}

Dans le but d'alléger l'exploration de l'effet d'incidence, nous avons scindé l'écoulement incident $\overrightarrow{V_{0}}$ en deux :

$$
\vec{V}_{0}=V_{0} \cos \alpha \vec{i}-V_{0} \sin \alpha \vec{j}
$$

$V_{0} \cos \alpha \vec{i}$ est un écoulement longitudinal non portant ; le calcul des singularités correspondantes $\left(\sigma_{1}\right)$ est effec- 
tué à partir de la partie carrée de $\left|A_{i j}\right| .\left(-V_{0} \sin \alpha \vec{j}\right)$ est un écoulement transversal portant qui nécessite pour son traitement la totalité de la matrice du système $(10)$ c'est-à-dire $\left|A_{i j} ! B_{i j}\right|$ les singularités correspondantes sont notées $\left(\sigma_{2}, \Gamma\right)$.

Chacun de ces écoulements est une solution du problème aux limites ; il en est de même d'une combinaison linéaire de ces solutions.

Ainsi, les vitesses tangentielles induites aux points de contrôle sont écrites comme combinaison linéaire des vitesses des écoulements longitudinal et transversal, soit :

$$
\begin{aligned}
& \vec{V}+\left(\vec{V}_{1}(M) / V_{0} \cos (\alpha)\right) V_{0} \cos \alpha \\
&+\left(\vec{V}_{2}(M) / V_{0} \sin \alpha\right) V_{0} \sin \alpha
\end{aligned}
$$$$
\text { où on } \mathrm{a} \text { : }
$$

$$
\begin{aligned}
& \vec{V}_{1} / V_{0} \cos \alpha=\sum_{j} \sigma_{j}^{(1)} X_{i j} \vec{i}+ \\
& \quad+\sum_{i} \sigma_{j}^{(1)} Y_{i j} \vec{j}+\sum_{j} \sigma_{j}^{(1)} z_{i j} \vec{k}
\end{aligned}
$$

où

$\vec{V}_{1}$ est la vitesse concernant l'écoulement longitudinal $\vec{V}_{2}$ est la vitesse concernant l'écoulement transversal $X X_{i j}, Y Y_{i j}, Z Z_{i j}$ : sont les composantes des vitesses $\sum_{k=1}^{24} F\left(v_{k}\right) x_{i k}^{\prime}{ }^{(1)} \ldots$ composantes de la vitesse induite $X_{\varphi}, Y_{\varphi}, Z_{\varphi}$, composantes de la vitesse tangentielle à la facette induite par les doublets de la quille.

L'équation de Bernoulli permet de calculer la pression aux points de contrôle :

$$
p / \sigma=C^{t e}-V^{2} / 2
$$

Par intégration de (18) sur l'ensemble carène-quille on a les forces et moments. Sous forme matricielle, le torseur de forces s'écrit :

$$
\left[\begin{array}{l}
\mathrm{F} \\
\overrightarrow{\mathrm{M}}
\end{array}\right]=\left[\begin{array}{lll}
\mathrm{Ex} & \mathrm{Fx} & \mathrm{Gx} \\
\mathrm{Ey} & \mathrm{Fy} & \mathrm{Gy} \\
\mathrm{Ez} & \mathrm{Fz} & \mathrm{Gz} \\
\mathrm{Emx} & \mathrm{Fmx} & \mathrm{Gmx} \\
\mathrm{Emy} & \mathrm{Fmy} & \mathrm{Gmy} \\
\mathrm{Emz} & \mathrm{Fmz} & \mathrm{Gmz}
\end{array}\right]\left[\begin{array}{c} 
\\
\\
\cos ^{2} \alpha \\
\sin ^{2} \alpha \\
\sin (2 \alpha)
\end{array}\right]
$$

Les coefficients $E, F$ et $G$ sont indépendants de l'incidence et sont calculés une fois pour toutes. L'ensemble des résultats sera exposé plus loin.

\section{Méthode expérimentale}

Les travaux expérimentaux avaient pour but principal de vérifier que le schéma mathématique adopté pour représenter l'écoulement correspondait aussi bien que possible avec la réalité. Pour vérifier le bien fondé du schéma mathématique, des essais, en soufflerie et en bassin des carènes, concernant des carènes et des quilles qui avaient subi le traitement mathématique choisi, ont été envisagés. La comparaison des résultats expérimentaux en soufflerie et des résultats obtenus par le calcul ayant donné des indications satisfaisantes quant à la corrélation schéma mathématique-expérience, les essais en soufflerie ont été poursuivis de manière systématique sur trois carènes données $(A, B, C)$, chaque carène étant étudié en présence de plusieurs quilles (Fig. 3).

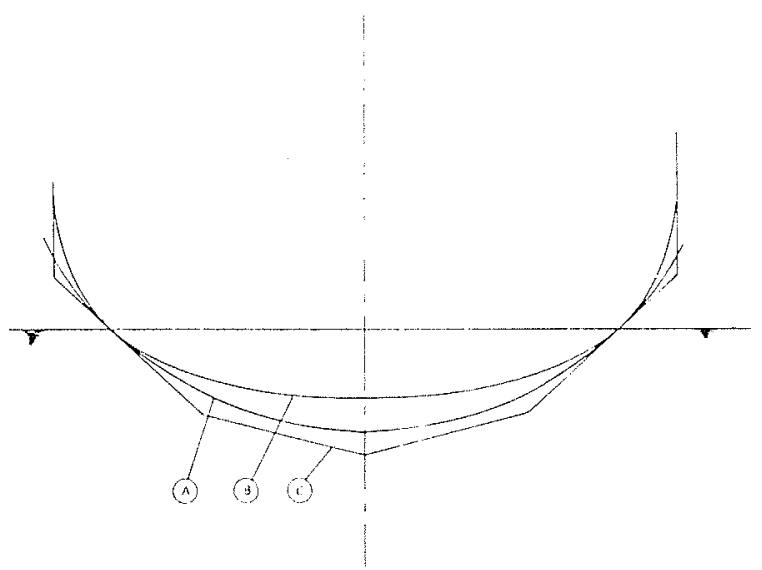

Figure 3 - Maître couple des carènes A, B, C.

Les essais en bassin nous ont permis de voir comment évoluent les résultats expérimentaux quand la carène est en présence de la surface libre. Pour ces essais nous avons repris comme modèle une des trois carènes étudiées en soufflerie, équipée d'une quille droite.

Pour chaque couple carène-quille étudié à une gîte donnée, nous avons mesuré, au moyen d'une balance à quatre composantes, solidaire de la quille deux forces et deux moments : traînée, portance, moment de roulis, moment de lacet.

Nous avons rencontré certaines difficultés dues à des décollements en soufflerie au voisinage de l'emplanture de la quille qui viennent perturber les mesures faites aux incidences supérieures à $5^{\circ}$ ou $6^{\circ}$. La cause de ces décollements semble être l'échelle réduite des modèles imposée par la taille de la soufflerie. Cette échelle augmente l'épaisseur relative de la couche limite sur la carene et favorise les décollements. Des essais ont été faits pour réduire l'épaisseur de la couche limite, qui se forme sur le plancher de la veine de mesure, au voisi- 
nage de la maquette, notamment par l'adjonction d'un deuxième plancher surélevé d'une distance représentant l'épaisseur moyenne calculée de la couche limite.

La comparaison des résultats obtenus avec le plancher simple et le double plancher nous a amené à revenir au simple plancher. En effet, les valeurs moyennes des efforts mesurés n'ont pas changé tandis que les perturbations instantanées étaient beaucoup plus grandes avec le double plancher. Par ailleurs, il n'était pas possible de limiter l'influence de la couche limite, développée sur la carène, par un anneau de garde autour de la quille, celuici aurait trop perturbé l'écoulement et entraîné des inconvénients plus importants que les avantages que l'on pouvait en attendre.

Une autre difficulté a été d'évaluer l'influence des parois de la veine de mesure sur les résultats. Cette influence a été évaluée en calculant les vitesses moyennes induites au niveau de la quille par les trois carènes images du modèle par rapport aux parois latérales et supérieure de la veine de mesure.

Les corrections calculées, dans le cas le plus défavorable où la quille a une gîte de $30^{\circ}$, sont de l'ordre d'un ou deux pour cent. Ces corrections, étant de l'ordre de grandeur des erreurs de mesure, n'ont pas été introduites dans les résultats.

\section{Résultats}

1 - L'évolution de la courbe de circulation en envergure sur la quille est "classique" c'est-à-dire du type de celle que l'on rencontre dans la théorie des surfaces portantes. La gite a pour effet de diminuer le maximum de circulation, alors que la flèche $y$ a peu d'influence (Fig. 4 et 5).

L'effet tridimensionnel est mis en évidence par l'évolution de la courbe des pressions en envergure, (Fig. 6).

2 - La comparaison des résultats obtenus en bassin, soufflerie et par la théorie montre une concordance assez bonne des méthodes théorique et expérimentale. L'écart sur les coefficients de portance est de l'ordre de 5-10\% (Fig. 7(a)). Il est normal de trouver un écart entre les courbes de trainée en bassin et la trainée induite, car la trainée de frottement n'est pas prise en compte par la théorie (Fig. 7(b)). La disparité sur les moments peut s'expliquer par le déplacement du point d'application des efforts expérimentaux. Ces comparaisons sont faites sur la carène $\mathrm{A}$ munie d'une quille de flèche nulle.

L'étude de l'effet du paramètre de gîte montre qu'il existe un écart notable sur le $C_{\nu} / \alpha$ aux faibles gites entre la théorie et l'expérience ; cet êcart est réduit à $10 \%$ aux fortes gîtes. Voir figure 8 . Cet écart est sans doute dû à la flèche $\left(\phi=45^{\circ}\right)$ qui pourrait introduire une modification de l'écoulement. Cela est confirmé sur les figures 9 et 10 où l'on a étudié la pente du $C_{y}$ en fonction de la flèche. Aux flèches faibles, l'estimation du $C_{y} / \alpha$ est satisfaisant (écart de $0,5 \%$ pour $\phi=0$, figure 10).

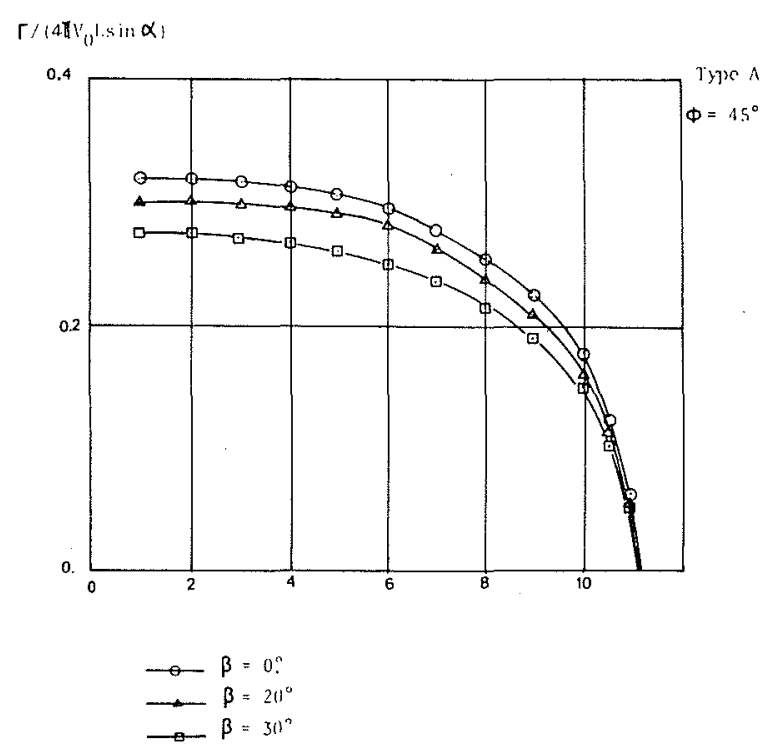

Figure 4 - Influence de la gite sur la distribution de circulation

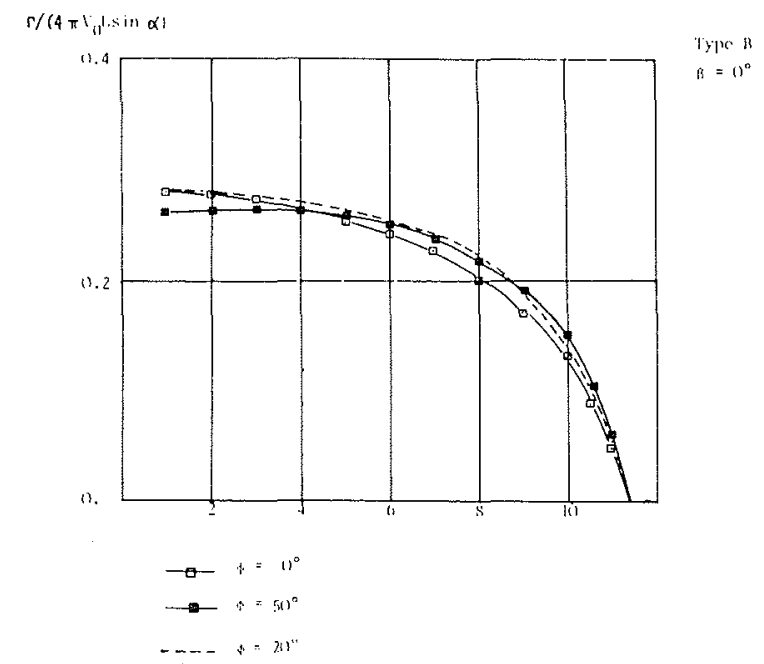

Figure 5 - Influence de la flèche sur la distribution de circulation

\section{3 - Influence des paramètres de l'écoulement (résultats expérimentaux)}

3.1 - La pente de la force de dérive $\left(C_{y} / \alpha\right)$ diminue lorsque l'angle de gîte $\beta$, augmente (Fig. 8 ). Les polaires relatives au couple carène quille $A Q_{1}$ font apparaître en soufflerie une trainée minimale pour des angles de gîte de $10^{\circ}$ et $20^{\circ}$ tant que l'incidence n'est pas trop grande (Fig. 11).

3.2 - L'influence de la flèche $\operatorname{sur}\left(C_{y} / \alpha\right)$ est faible et de plus est indépendante de l'angle de gite $\beta$ (Fig. 9 et $10)$. Le tracé des polaires n'est pratiquement pas modifié par l'angle de flèche aux grandes incidences ; il semble qu'aux faibles incidences la flèche de $15^{\circ}$ permet d'avoir une trainée réduite (Fig. 12). 

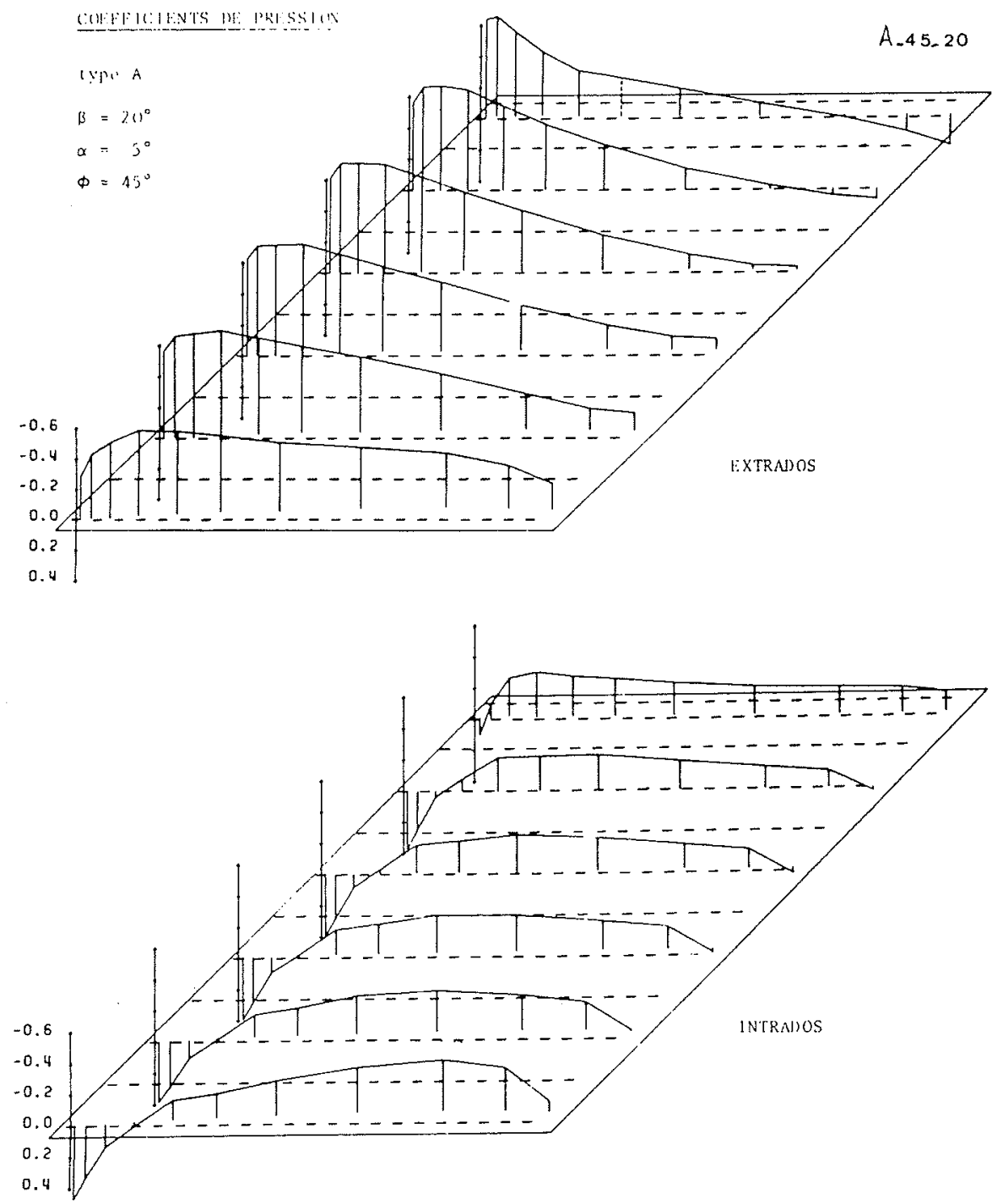

Figure 6
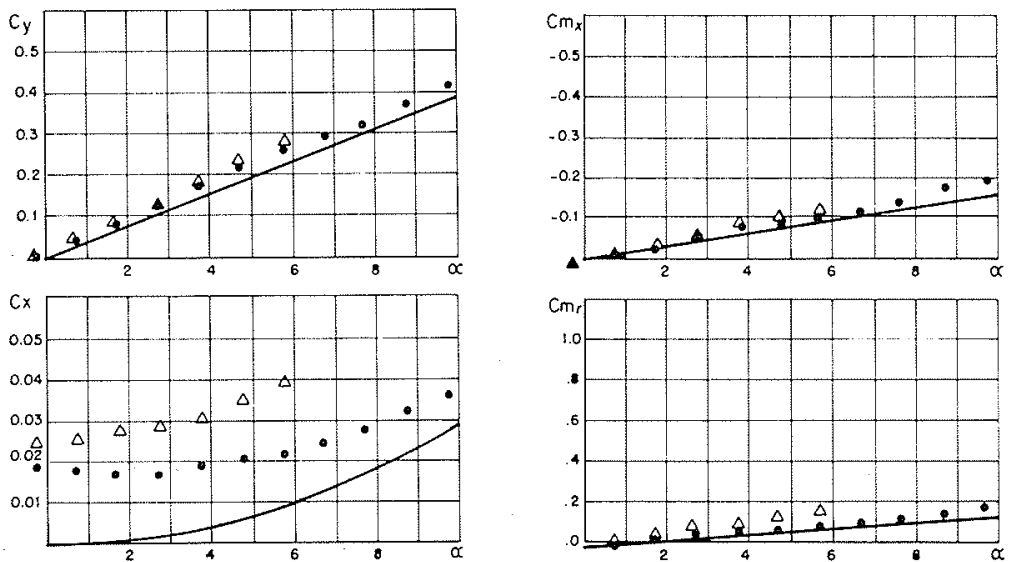

- Soufflerie

$\triangle$ Bassin $(F r=0.32)$

-Théorie

$\mathrm{H} / \mathrm{C}=.37$

$L / C=5.34$

$T / C=.889$ 


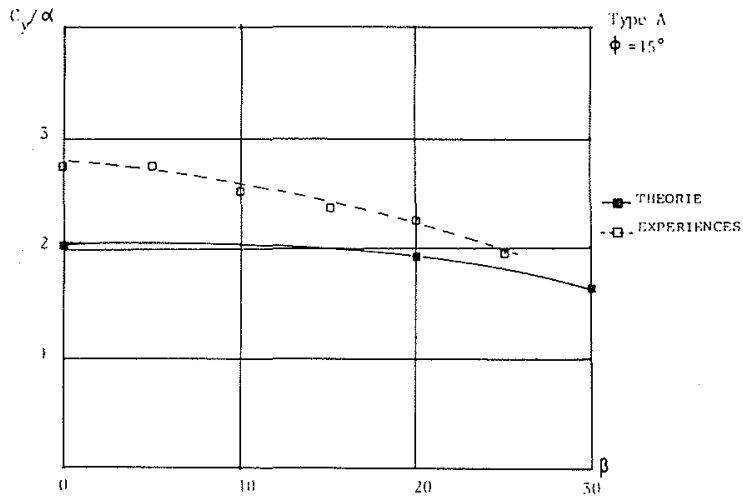

Figure $8-C_{y} / \alpha$ quille seule

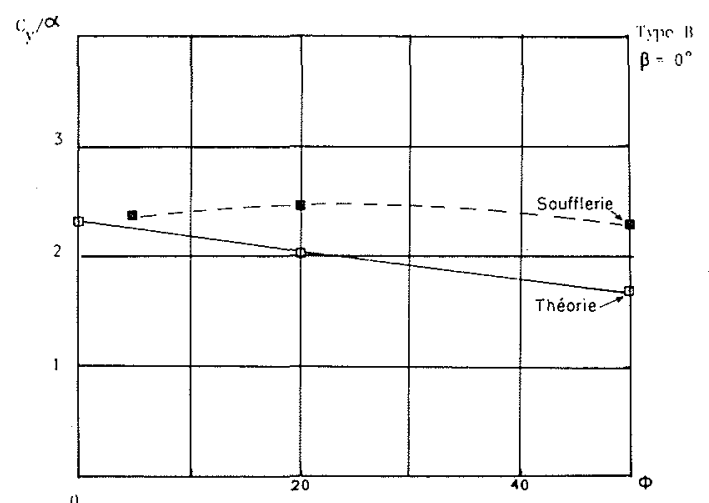

Figure $9-C_{y} / \alpha$ quille seule

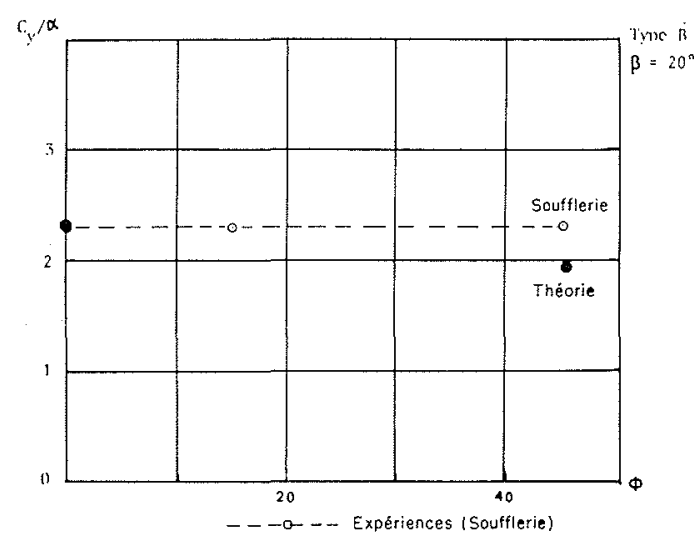

Figure $10-C_{y} / \alpha$ quille seule

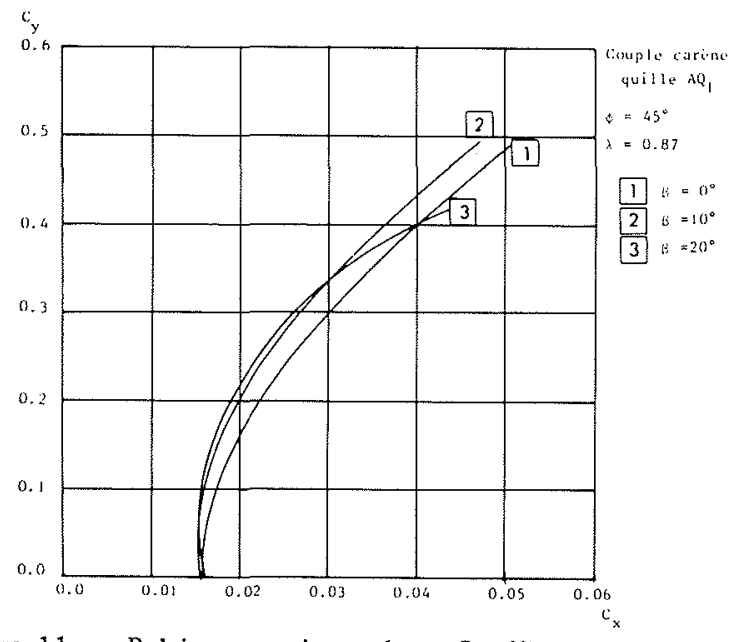

Figure 11 - Polaires experimentales - Soufflerie influence de la gite

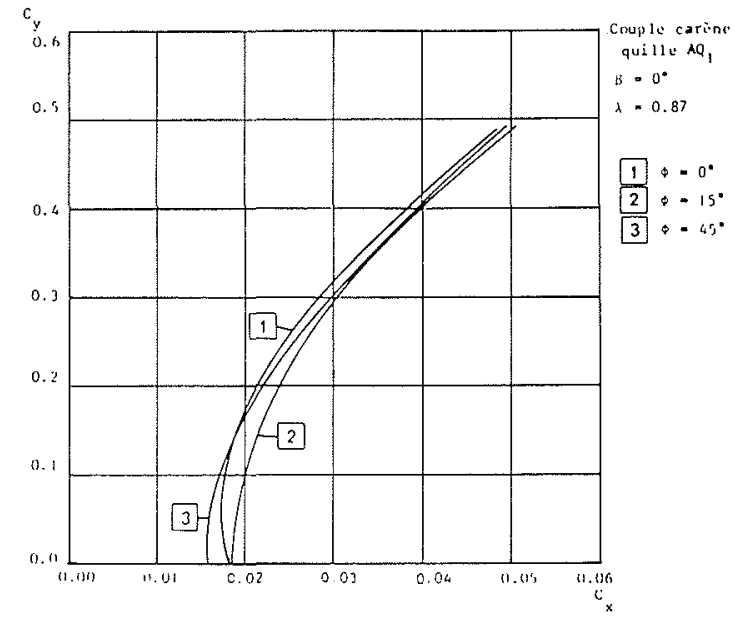

Figure 12 - Polaires expérimentales - Soufflerie influence de la flèche

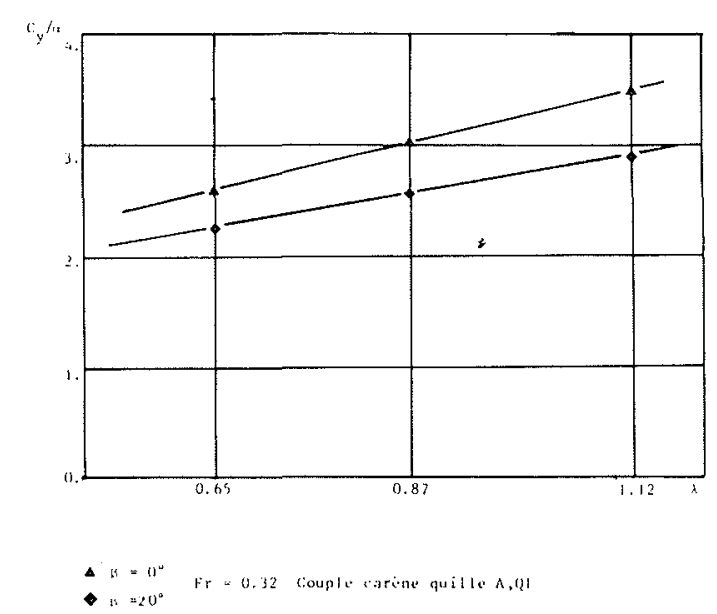

Figure 13 - Influence de l'allongement sur la pente de la portance.

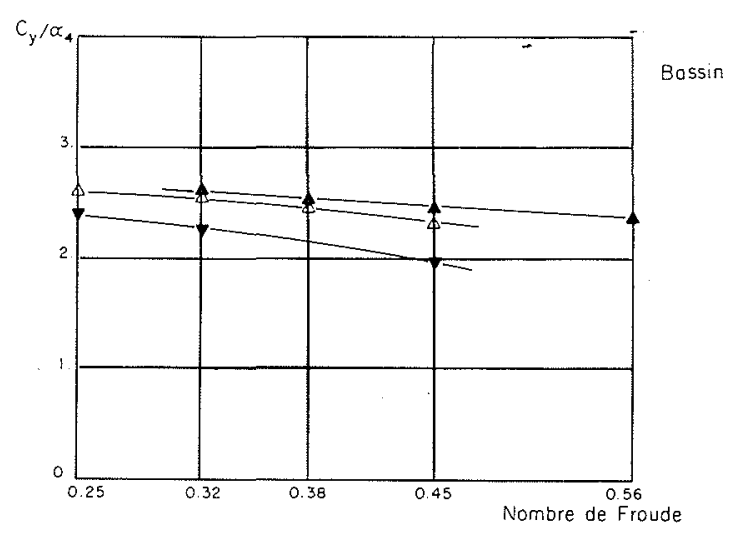

$\begin{array}{lll}\beta & =0^{\circ} \quad \lambda=0.65 \\ \beta & \lambda=0.07\end{array}$

$\left.\triangle \beta=20^{\circ} \lambda=0.87\right\}$ Couple carène quille $A, Q 1$

$\nabla \beta=20^{\circ} \lambda=0.65$

Figure 14 


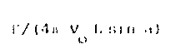

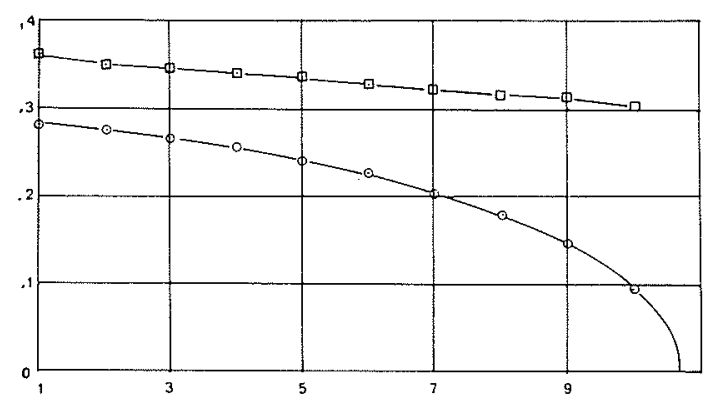

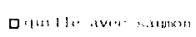

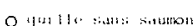

Figure 15 - Distributions de circulation d'une quille avec et sans saumon.
3.4 - Dans le cas d'un angle de gîte nulle la pente de la portance reste sensiblement constante quand le nombre de Froude varie de 0,32 à 0,56 (Fig. 14). Si la carène est en gite, la pente $\left(C_{v} / \alpha\right)$ varie peu mais on observe un décalage de l'axe de portance nulle, décalage croissant avec le nombre de Froude. La trainée expérimentale est très peu influencée par le nombre de Froude pour des angles de gîte nulle.

\section{Influence du saumon (résultats théoriques)}

L'influence du saumon a été mis en évidence sur le Pen Duick III. La distribution de circulation est modifiée par le saumon (Fig. 15) ; l'écart entre les deux distributions, avec et sans saumon, se traduit par un écart considérable sur la portance et la trainée induite. De même, les $C M_{X}$ et $C M_{Z}$ sont modifiés dans des proportions comparables (Fig. 16).

\section{Conclusion}
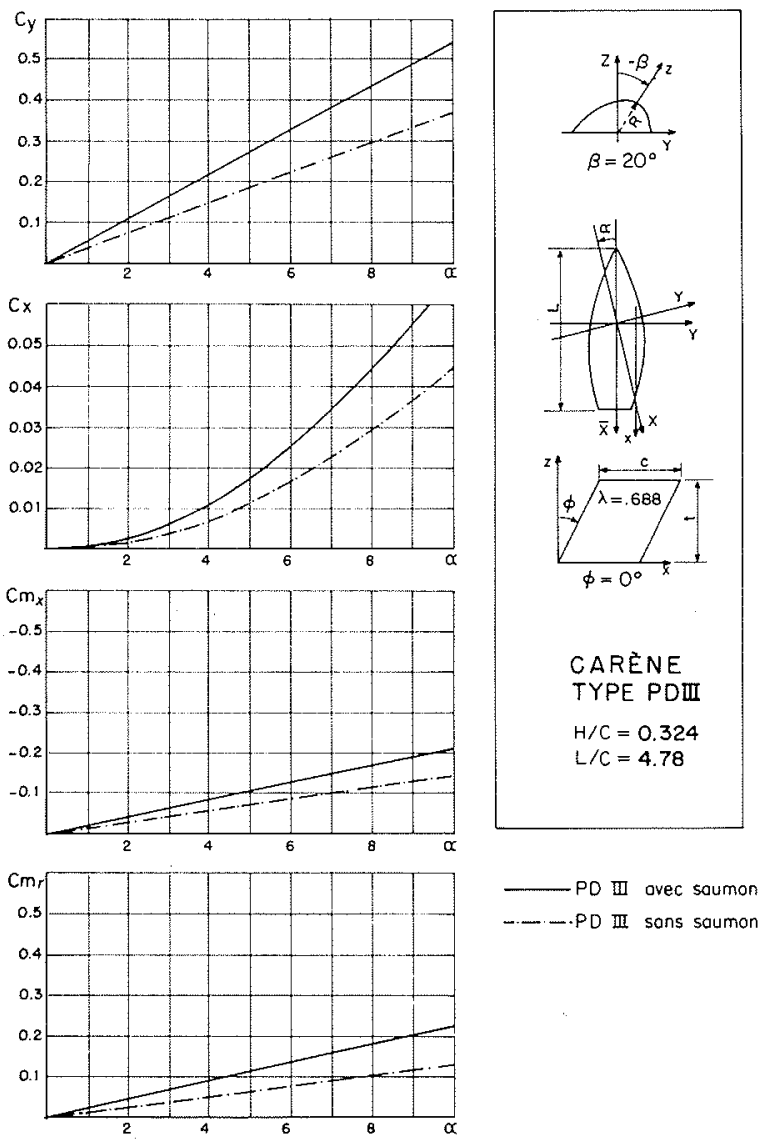

-._. .PO III sans saumon

Figure 16 -

3.3 - Lorsque l'allongement croît la pente de la force de derive augmente. Lorsque la gite augmente, la croissance du $C_{y} / \alpha$ est ralentie (Fig. 13).
Le bon accord des résultats théoriques avec les essais expérimentaux est encourageant pour la suite du travail et assure le bien fondé de la méthode adoptée.

Il est possible d'appliquer le programme pour comparer deux ou plusieurs couples carène-quille donnés et en tirer des renseignements sur les performances hydrodynamiques des ailerons utilisés.

On a pu constater que les mesures de portance et trainée en bassin sont en accord avec celles de la soufde négliger la condition de surface libre n'a donc pas été une hypothèse trop restrictive ; cela se comprend dans la mesure où l'influence des ondes de la surface libre diminue très rapidement dès que l'on évolue en profondeur. Il est évident que cette hypothèse entraînerait des résultats erronés sur la carène qui coupe la surface libre.

A moyen terme, nous envisageons de généraliser la méthode dans le cas d'une carène portante et d'introduire le pilonnement et le tangage ; à plus long terme il serait intéressant d'introduire le nombre de Froude dans les calculs et d'étudier dans sa généralité le problème instationnaire mais ce problème n'a pas encore trouvé de solution, même pour un bateau classique. flerie pour des nombres de Froude de 0,3 à 0,32 . Le fait
Les auteurs tiennent à exprimer leurs remerciements au Professeur Patrice Sulmont qui a patronné ces recherches et à Monsieur T.S. Luu, Directeur de recherches au LIMSI, pour ses conseils éclairés. 


\section{Bibliographie}

[1] LUU J.S., COULMY G., CORNIGLION J. - Calcul non linéaire de l'écoulement à potentiel autour d'une aile d'envergure finie de forme arbitraire. A.T.M.A. 1971.

[2] HESS J.L., SMITH A.M.O. - Calculation of non lifting potential flow about arbitrary three dimensional bodies. Douglas Aircraft Company - 1962

[3] HESS J.L. - The problem of three-dimensional lifting potential flow and its solution by means of surface singularity distribution. Theoretical Aerodynamics Section -
Douglas Aircraft Company - 1970.

[4] MALAVARD - Théorie et méthodes de calcul relatives aux lignes et surfaces portantes. Cours à la Faculté des Sciences de Paris.

[5] LUU J.S., COULMY G., CORNIGLION J. - Techniques des effets élémentaires de singularités dans la résolution des problèmes d'hydro et d'aérodynamique. A.T.M.A. 1969.

[6] RAVILLY E. - Essais en soufflerie des ailerons expérimentaux de Pen Duick III. Annales E.N.S.M. 1968.

[7] SULMONT P., JACQUOT C., RAJAONA D. - Etudes de carènes de voiliers. Rapport D.G.R.S.T. Avril 1976 Contrat n $n^{\circ}$ 73.7.1769 - Annexe 1 - Annexe 3.

\section{Discussion}

M. le Président remercie MM. JACQUOT et RAJOANA pour leur intéressant exposé et passe la parole à l'auditoire qui, malheureusement, s'est réduit en raison de l'heure tardive.

Peut-on évaluer, éventuellement à partir de l'expérimentation, l'importance de l'erreur attendue du fait de la non prise en compte de la déformation de la surface libre ? demande M. DODU.

$\mathrm{Au}$ cours des essais en bassin, répond M. RAJOANA, les effets de la surface libre sur la quille sont pratiquement négligeables ; je ne connais pas son influence sur la carène. Dans nos essais, la quille avait 10 centimètres d'envergure et la carène un tirant d'eau de 2 à 3 centimètres; les vitesses de transiation allaient jusqu'à $1,80 \mathrm{~m} / \mathrm{s}$.

M. GUEVEL précise : la prise en compte de la déformation de la surface libre revient à in troduire le potentiel du champ de vagues d'accompagnement. Or, pour les nombres de Froude usuels, celui-ci s'amortit assez rapidement en profondeur. Aussi, je ne pense pas que les effets de surface libre se manifestent d'une manière déterminante au niveau de la quille d'un voilier ; s'il n'en était pas ainsi, les calculs deviendraient extrêmement compliqués et nécessiteraient des temps de traitement sur ordinateur qui ne permettraient pas d'exploiter les programmes de calcul pour les besoins de la pratique.

A la suite de nombreuses discussions avec MM. JACQUOT et RAJAONA, je crois savoir que ceux-ci ont lintention de justifier théoriquement, du moins pour quelques cas types, le bien fondé de l'approximation qui consiste à négliger la déformation de la surface libre.

\section{Clôture de la Session. Allocution de M. P. WILLM}

En l'absence d'autre intervention, $M$. le Président conclut en ces termes:

Je remercie encore les Conférenciers qui m'ont beaucoup instruit sur la façon d'aborder les problèmes de voiliers que je pensais, jusqu'à présent, du domaine de l'architecture navale traditionnelle; leurs modèles sont accompagnés d'une approche théorique, qui pour l'instant est limitée, mais qu'il paraît intéressant d'encourager pour l'avenir. 